\title{
Myocarditis confirmed by biopsy presenting as acute myocardial infarction
}

\author{
MARIA ROSA COSTANZO-NORDIN, * JOHN B O’CONNELL,^ RAMIAH SUBRAMANIAN, $†$

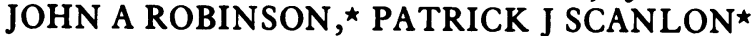 \\ From the Departments of ${ }^{\star}$ Medicine (Sections of Cardiology and Immunology) and †Pathology, Loyola University \\ Medical Center, Maywood, Illinois, USA
}

SUMMARY Two cases of acute myocardial infarction occurred in association with myocarditis, which was confirmed by biopsy. The first patient suffered an anteroseptal and the second patient an inferior wall myocardial infarction shortly after an acute viral illness. In both patients, coronary angiography showed normal coronary arteries, and right ventricular endomyocardial biopsy confirmed myocarditis. Histological abnormalities attributable to ischaemic heart disease were absent. The first patient's condition became stable after immunosuppressive treatment. Myocarditis resolved spontaneously within three months in the second patient. Coronary artery spasm and myocardial involvement with a systemic disease were unlikely.

Endomyocardial biopsy in patients with acute myocardial infarction and normal coronary arteries may be useful in identifying myocarditis associated with myocardial necrosis. Myocarditis in acute myocardial infarction in the absence of coronary artery obstruction has not previously been documented during life.

Systemic viral infection is often complicated by acute myocarditis in man, but the actual pathophysiology of the resulting myocardial dysfunction is unclear. ${ }^{1}$ Animal models of enteroviral myocarditis have elucidated the pathogenesis of human myocardial inflammation. ${ }^{2}$ Murine Coxsackie virus B1 and B4 infection produce not only extensive myocardial inflammation but also coronary arteritis, ${ }^{3}$ localised myocardial necrosis, and, occasionally, ventricular aneurysms, indistinguishable from those following myocardial infarction induced by epicardial coronary artery obstruction. ${ }^{45}$ The presence of myocarditis in patients who suffered myocardial infarction despite the absence of coronary artery disease has been found at necropsy. ${ }^{6}$ The occurrence of myocarditis in this setting has not been previously documented during life, and a causal relation between the two entities remains controversial. It is estimated that $1 \%$ of all myocardial infarctions ${ }^{7}$ and up to $17 \%$ of myocardial infarctions occurring in younger patients occur with-

Requests for reprints to Dr Maria Rosa Costanzo-Nordin, Loyola University Medical Center, 2160 South First Avenue, Maywood, Illinois 60153, USA.

Accepted for publication 23 August 1984 out demonstrable coronary artery disease ${ }^{8}$ We report two cases of myocardial infarction occurring in association with myocarditis confirmed by biopsy.

\section{Patients and methods}

\section{CASE REPORTS}

Case 1

A 56 year old man had long standing rheumatoid arthritis, without extra-articular manifestations, which was chronically treated with low dose corticosteroids. Shortly after a viral syndrome with high fever, sore throat, myalgias, and anorexia he developed severe pressure-like substernal chest pain radiating to the left jaw and lasting several hours. Serial electrocardiograms showed the evolution of extensive anterolateral injury and an old inferior wall myocardial infarction. Cardiac catheterisation, performed two weeks after the acute event, showed a large apical aneurysm with filling defects suggestive of mural thrombi. The coronary arteries were essentially normal. Four months later, in view of persisting symptomatic congestive heart failure, he was referred to determine possible myocarditis.

On physical examination his blood pressure was 
$114 / 78 \mathrm{~mm} \mathrm{Hg}$, pulse rate 88 beats $/ \mathrm{min}$, respiration rate $24 / \mathrm{min}$, and temperature $36.8^{\circ} \mathrm{C}$. Cardiac examination showed a diffuse apical impulse with a summation gallop. Rales were heard in both lung bases, and pedal oedema was present. An electrocardiogram showed the residual changes of an extensive anterolateral infarction with persistent ST elevation in V2-V6 as well as the old inferior wall myocardial infarction (Fig. 1a). His initial laboratory tests showed a normal
Fig. 1 Case 1: (a) electrocardiogram showing extensive anterolateral infarction with ST elevation in leads V2-V6 and an old inferior wall myocardial infarction. (b) $A$ gallium ${ }^{67}$ scan in the anterior and $45^{\circ}$ left anterior oblique projection showing myocardial uptake (arrows). (c)

Photomicrograph of endomyocardial biopsy specimen showing interstitial lymphomononuclear cell infiltration (arrow) and myocyte necrosis (haematoxylin and eosin, original magnification $\times 200$ ).

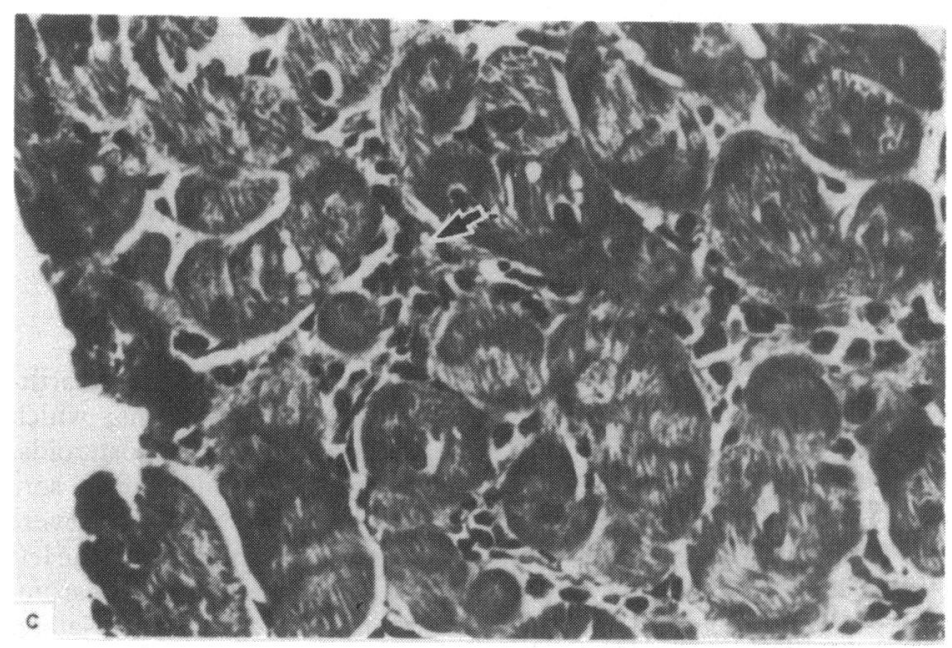

differential blood count, serum protein electrophoresis, and concentration of complement. The rheumatoid factor was positive at a titre of 1/1280. Antinuclear factor was absent. Coxsackie B1-B6 serum antibody titres were $<1 / 8$. A chest radiograph showed mild cardiomegaly. Autologous indium ${ }^{111}$ leucocyte and gallium ${ }^{67}$ scans showed pronounced myocardial uptake (Fig. 1b). Radionuclide ejection fraction was $17 \%$ with the apical aneurysm. 

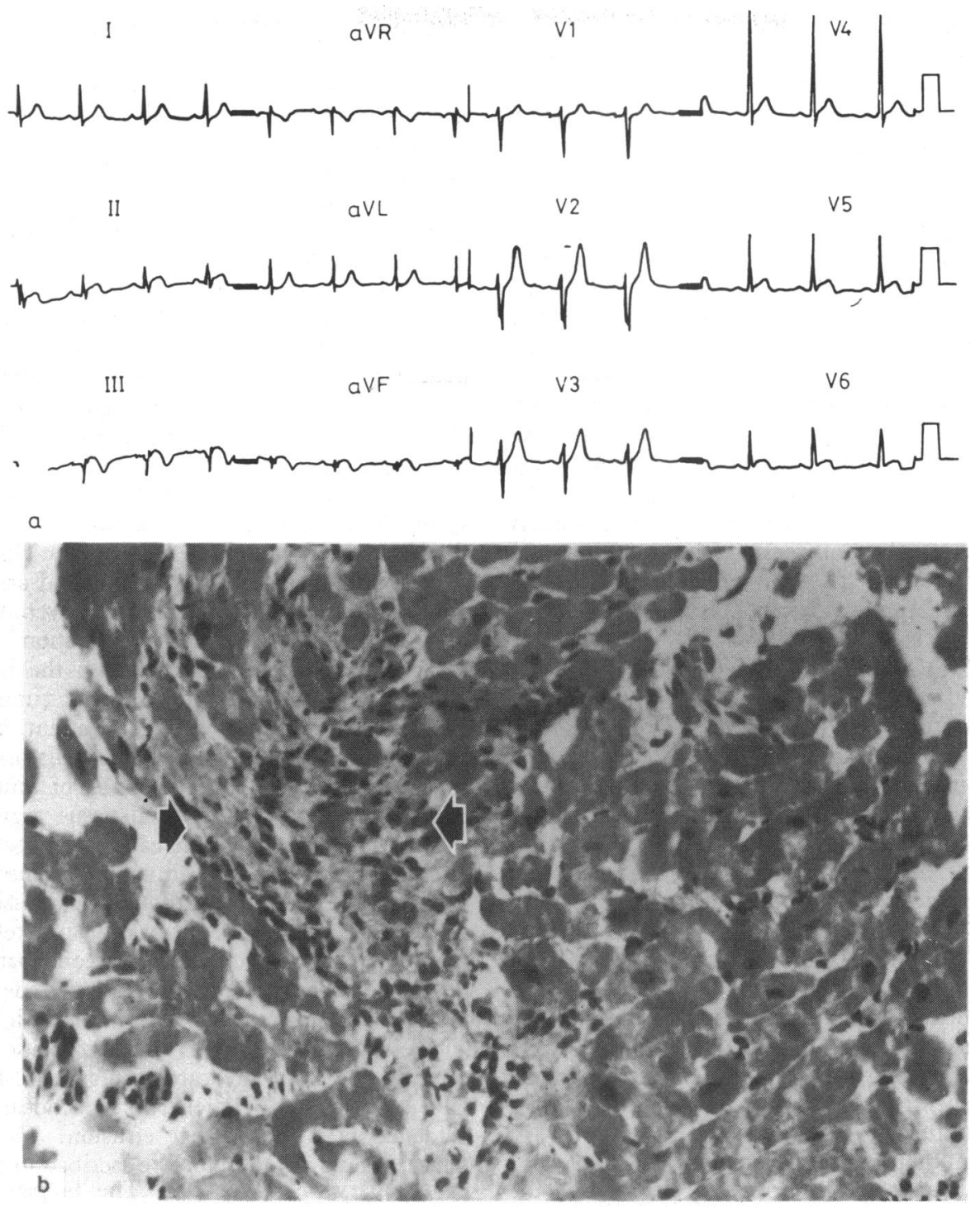

Fig. 2 Case 2: (a) electrocardiogram showing changes of an acute inferior wall myocardial infarction and (b) photomicrograph of endomyocardial biopsy specimen showing lymphomononuclear infiltration, myocyte necrosis, and fibrosis (arrows) (haematoxylin and eosin, original magnification $\times 100$ ).

A right ventricular endomyocardial biopsy showed focal perivascular and myocardial lymphocytic infiltration with myocyte necrosis, consistent with active myocarditis. No interstitial oedema, polymorphonuclear leucocyte infiltration, or coagulation necrosis of the myocytes was seen (Fig. 1c).

Immunofluorescence showed no immunoglobulin or complement deposition in the heart. Viral cultures and electron microscopy failed to demonstrate virus in the myocardium. The prednisone dosage was increased and azathioprine was added. The patient's condition was stable 16 months after the event.

\section{Case 2}

A 17 year old previously healthy white boy presented with a one week history of sore throat, low grade fever, sporadic daily palpitation, and pressure-like non-pleuritic chest pain radiating to his left shoulder. On physical examination his blood pressure was 110/ $68 \mathrm{~mm} \mathrm{Hg}$, heart rate 71 beats $/ \mathrm{min}$, respiration rate 
regular at $16 / \mathrm{min}$, and temperature $38.2^{\circ} \mathrm{C}$. The throat was erythematous. Cardiac examination showed a normal apical impulse and heart sounds, a flow murmur, and physiological third heart sound. $\mathrm{He}$ had no pericardial friction rub or hepatosplenomegaly.

A telemetry rhythm strip obtained on admission showed a short episode of accelerated idioventricular rhythm. Laboratory values showed a mild leucocytosis with left shift, an erythrocyte sedimentation rate of $56 \mathrm{~mm}$ in the first hour, and negative blood and throat cultures. Ebstein-Barr and Coxsackie viral titres, and antistreptolysin $O$ titre were within normal range. His peak creatinine phosphokinase activity was $857 \mathrm{IU} / 1$ with a strongly positive $\mathrm{MB}$ band, serum aspartate aminotransferase activity $150 \mathrm{IU} / 1$, and lactate dehydrogenase activity $558 \mathrm{IU} / 1$. His cardiac enzyme values gradually returned to normal over three days showing the classic evolutionary pattern of an acute myocardial infarction. Liver function was normal.

The electrocardiogram showed ST elevation in leads II, III, aVF, and V5-V6 with T wave inversion and $Q$ wave formation in those leads consistent with inferior infarction (Fig. 2a). His condition stabilised, and cardiac catheterisation performed one week later showed normal coronary arteries and left ventricular function. Radionuclide left ventricular ejection fraction was $64 \%$. An echocardiogram showed no pericardial effusion. An autologous indium ${ }^{111}$ leucocyte scan showed myocardial uptake. A 24 hour electrocardiographic recording showed episodes of sinus tachycardia, multiple unifocal ventricular extrasystoles, and short runs of ventricular bigeminy.

Right ventricular endomyocardial biopsy showed focal lymphocytic infiltrates and associated myofibre necrosis compatible with active myocarditis (Fig. 2b). After an uneventful clinical course, he was discharged in good condition.

Myocardial biopsy was repeated three months later to confirm the resolution of myocarditis before the patient was allowed to resume his usual school and sporting activities. Irregular myocyte hypertrophy, focal myofibril loss, and increased interstitial fibrosis were present. No inflammatory infiltrates were detected. The histological changes were considered to represent healed myocarditis. A follow up electrocardiogram showed persistent $T$ wave inversion in the inferolateral leads. To date the patient is in good health and performs his work without limitations.

\section{Discussion}

These two patients had clinical and electrocardiographic evidence of myocardial infarction in the clinical setting of acute myocarditis. Non-invasive imaging with indium ${ }^{111}$ labelled leucocytes and gallium ${ }^{679}$ suggested the presence of myocardial inflammation, which was confirmed by endomyocardial biopsy. The diagnosis of myocarditis was based on the presence of 5 lymphocytes per high power field and associated myocardial necrosis. The foci of active myocarditis identifying unsuspected myocarditis in association from the infarcted area, as defined by the electrocardiogram, emphasising the efficacy of this technique in identifying unsuspected myocarditis in association with unexplained myocardial necrosis. No histological changes suggesting ischaemic heart disease were found. Lymphocytic infiltration and myofibre necrosis were detected in the first patient four months after the acute myocardial infarction, and it is therefore highly unlikely that they represent the histological manifestations of myocardial infarction by themselves. In the first patient, viral infection was the most likely cause of the febrile illness. The chronic corticosteroid treatment for his rheumatoid arthritis may possibly account for the unusually severe myocardial involvement. Myocardial infarction due to rheumatoid coronary vasculitis, in the presence of extensive extra-articular disease and cutaneous vasculitis, has been reported. ${ }^{10}$ Our patient, in contrast, had no evidence of extra-articular disease, and the absence of bound immunoglobulin or immune complex deposition in myocardial tissue argues against rheumatoid arthritis as the cause of myocarditis and infarction. This patient had no symptoms suggesting coronary artery spasm. Although this possibility cannot be eliminated, this entity is an unlikely cause of his myocardial infarction. The second patient had a viral syndrome with sore throat, low grade fever, and myalgias. He presented with non-pleuritic chest pain and electrocardiographic and enzymatic evolution typical of an acute myocardial infarction. He did not have a pericardial friction rub or echocardiographic evidence of a pericardial effusion. These abnormalities could not therefore be ascribed to pericarditis or coronary artery disease. The biopsy confirmed active myocarditis as the likely cause of the myocardial necrosis.

The two patients underwent coronary angiography within two weeks of acute myocardial infarction. The possibility of an intracoronary thrombus resulting in acute myocardial infarction and resolving without residual coronary obstruction cannot be excluded. Neither patient, however, had a history of chest trauma, heavy exertion, or excessive smoking, and thus in situ coronary thrombus formation is an unlikely cause of their myocardial infarction.

Careful clinicopathological correlations at necropsy have shown that viral myocarditis has a wide spectrum of clinical presentations. Subsequent to the systemic manifestations of a viral syndrome (fever, upper 
respiratory infection, myalgias, arthralgias, gastrointestinal complaints) patients with myocarditis may be asymptomatic or develop pericarditis, arrhythmias, progressive circulatory failure or precordial pain, and electrocardiographic changes. ${ }^{211}$ Symptoms and signs, including exertional non-pleuritic chest pain, increased enzyme activity, and the appearance of ST elevation, $T$ wave inversion, and abnormal $Q$ waves in the electrocardiogram indistinguishable from myocardial infarction have also been observed. ${ }^{12}$ Nevertheless, histological documentation during life has not previously been reported.

Serological evidence of Coxsackie B virus infection in man has been identified in $10-18 \%$ of patients with documented myocardial infarction. These data are not consistent and vary with the patient population, assay method, and sampling times. ${ }^{1314}$ The confusion surrounding the association of myocardial infarction with myocarditis is perpetuated by the lack of unequivocal documentation of the latter process after acute myocardial infarction occurring in the setting of acute viral infection.

In the experimental model, a necrotising transmural myocarditis has been produced in mice infected with Coxsackie virus B1 or B4. Several weeks after infection, $10 \%$ of the animals had aneurysmal dilatation of the left ventricle. Myocardial thinning, necrosis, and normal coronary vessels were found in the affected areas. ${ }^{5}$ These findings strongly suggest that some ventricular aneurysms may represent complications of a preceding unrecognised necrotising myocarditis and may parallel similar findings in man.

Based on our findings, selected patients with clinical, electrocardiographic, and laboratory evidence of myocardial infarction but normal coronary arteries, in whom coronary artery spasm or a resolved intracoronary thrombus are unlikely, may be considered for inflammation-avid isotopic imaging and endomyocardial biopsy to identify myocarditis associated with myocardial necrosis. After resolution of the acute event, if inflammation and cardiac dysfunction persist, treatment designed to suppress inflammatory response may be appropriate. ${ }^{15}$

\section{References}

1 Abelmann WH. Virus and the heart. Circulation 1971; 44: 950-6.

2 Woodruff JF. Viral myocarditis. Am F Pathol 1980; 101: 427-79.

3 Burch GE. The coronary arteries in active viral cardiomyopathies. Postgrad Med F 1975; 51: 311-24.

4 El-Khatib MR, Chason JL, Lerner AM. Ventricular aneurysms complicating Coxsackie virus Group B, Types 1 and 4 murine myocarditis. Circulation 1979; 59: 412-6.

5 Khatib R, Chason JL, Lerner AM. A mouse model of transmural myocardial necrosis due to Coxsackie virus B4. Observations over 12 months. Intervirology 1982; 18: 197-202.

6 Saffitz JE, Schwartz DJ, Southworth W, et al. Coxsackie viral myocarditis causing transmural right and left ventricular infarction without coronary narrowing. $A m \mathcal{F}$ Cardiol 1983; 52: 644-7.

7 Cheitlin M, McAllister HA, DeCastro CM. Myocardial infarction without atherosclerosis. $\mathcal{F A M A} 1975 ; 231$ : 951-9.

8 Glover MU, Kuber MT, Warren SE, Vieweg WVR. Myocardial infarction before age 36: risk factor and arteriographic analysis. Am $\mathcal{Y}$ Cardiol 1982; 49: 1600-3.

9 Robinson JA, O'Connell J, Henkin RE, Gunnar RM. Gallium $^{-67}$ imaging in cardiomyopathy. Ann Intern Med 1979; 90: 198-9.

10 Voyles WF, Searles RP, Bankhurst AD. Myocardial infarction caused by rheumatoid vasculitis. Arthritis Rheum 1980; 23: 860-3.

11 Gardiner AJS, Short D. Four faces of acute myopericarditis. Br Heart $\mathcal{F}$ 1973; 35: 433-42.

12 Take $\mathbf{M}$, Sekiguchi $\mathbf{M}$, Hiroe $\mathbf{M}$, Horosawa K. Early clinical profiles of cases with histopatholgically proven acute idiopathic myocarditis and a proposal for diagnostic criteria. Fpn Circ F 1981; 45: 1415-20.

13 Griffiths PD, Hannington C, Booth JC. Coxsackie B virus infections and myocardial infarction. Lancet 1980; i: $1387-9$.

14 Lau RCH. Coxsackie B virus infection in acute myocardial infarction and adult heart disease. Med $\mathcal{F}$ Aust 1982; 2: 520-2.

15 O'Connell JB, Robinson JA, Henkin RE, Gunnar RM. Immunosuppressive therapy in patients with congestive cardiomyopathy and myocardial uptake of Gallium-67. Circulation 1981; 64: 780-6. 